\title{
Effective task scheduling algorithm in cloud computing with quality of service alert bees and grey wolf optimization
}

\author{
Nidhi Bansal', Ajay Kumar Singh ${ }^{2}$ \\ ${ }^{1}$ Department of Computer Science and Engineering Department, \\ PhD student of AKTU (Formerly Uttar Pradesh Technical University), Lucknow, India \\ ${ }^{2}$ Department of Computer Science and Engineering Department, \\ Faculty of Meerut Institute of Engineering \& Technology, Meerut, India \\ Affiliation with AKTU (Formerly Uttar Pradesh Technical University), Lucknow, India
}

\section{Article Info \\ Article history: \\ Revised Oct 25, 2021 \\ Keywords: \\ Cloud computing \\ Cost \\ Grey wolf \\ Honey bees \\ Task scheduling}

Received Jun 19, 2021

Accepted Nov 30, 2021

\begin{abstract}
Quality-based services are an indicative factor in providing a meaningful measure. These measures allow labeling for upcoming targets with a numerical comparison with a valid mathematical proof of reading and publications. By obtaining valid designs, organizations put this measure into the flow of technology development operations to generate higher profits. Since the conditions were met from the inception of cloud computing technology, the market was captured heavily by providing support through cloud computing. With the increase in the use of cloud computing, the complexity of data has also increased greatly. Applying natural theory to cloud technology makes it a fruit cream. Natural methods often come true, because survival depends on the live events and happenings, so using it in real life as well as any communication within technology will always be reliable. The numerical results are also showing a better value by comparing the optimization method. Finally, the paper introduces an adaptation theory with effective cloudsim coding of honey bees and grey wolf in conjunction with their natural life cycle for solving task scheduling problems. Using adapted bees improved the results by $50 \%$ compared with the original bees and secondly by honeybees and grey wolf improved $60 \%$.
\end{abstract}

This is an open access article under the CC BY-SA license.

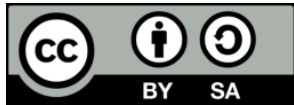

\section{Corresponding Author:}

Nidhi Bansal

Department of Computer Science and Engineering Department

$\mathrm{PhD}$ student of AKTU (Formerly Uttar Pradesh Technical University), Lucknow, India

Email: nidhi18jul@gmail.com

\section{INTRODUCTION}

Cloud computing is doing very well in the direction of technology, so all experts are developing new innovation ideas using a combination of natural activity in the cloud environment. By providing an advantage in developing production and organization, experts are using cloud technologies in the education sector to make people skillful and valuable. This will also reduce the unemployment rate. By this, most people are receiving their education at home as well. Cloud computing is evolving through researchers and deploying its nature over time. The requirements are reaching towards the richness of effective techniques and resource pooling facility by deriving analysis on big data derived from databases. It starts with small instruments and goes into elephant shape with its richness. As content is increasing regularly, the technology will have to be updated every period. Every skill must reach every needy door.

To define task scheduling in cloud computing, it is used to select resources for execution with minimal wait and time. Mostly it is used to reduce revert time so that machines can work with full efficiency 
even with multitasking. With the vast expansion of technology, the cloud is still alive to achieve diffusion in computing with many challenges. Figure 1 is showing the basics of task scheduling in cloud computing where six tasks are working parallel to the left and four virtual machines are available to execute with the latest optimized scheduling algorithms.

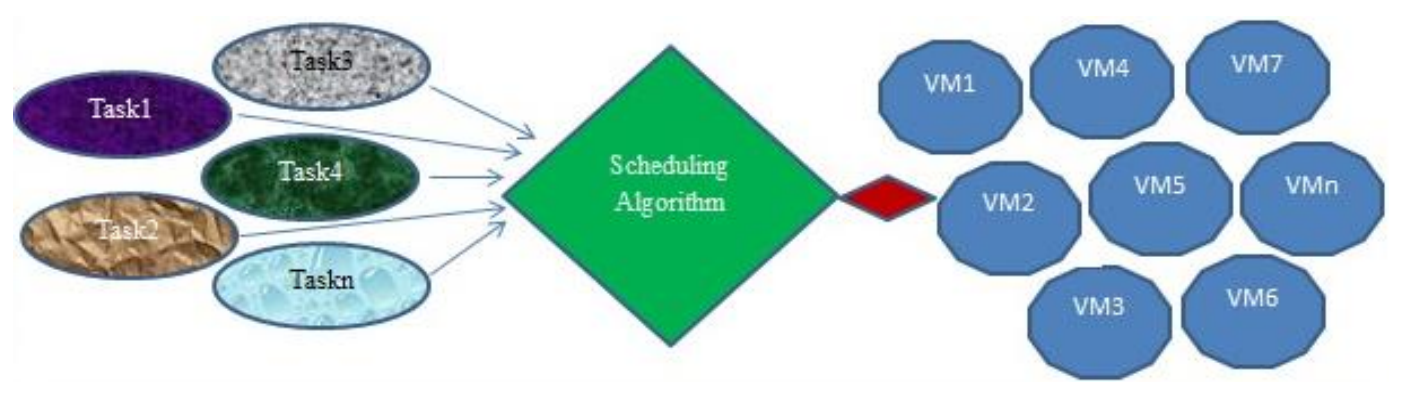

Figure 1. Task scheduling in cloud computing

In the last years, most of researchers point to solve cloud computing challenges to develop the cloud performance. These challenges are and not limited to, security, power consumption, latency, resource management, and task scheduling. In this paper we will focused on task scheduling problem. Many natural adapted methods have been in great use in the technological world due to their high ranking. In particular, some examples of them are particle swarm optimization (PSO), ant colony optimization (ACO), Simulation annealing (SA) and genetic algorithms (GA) very effectively not only among researchers from computer backgrounds, but also among various community scientists. Natural based algorithms are also performing well in education, development and production. This technique is well-known because its useful features are: easy to understand, expandable convenience as per the new version of the technology, self-generated function capability and failure average.

Methods of remote sensing are also in the subject, to get a beautiful digital world. Using these iconic things, the world can very easily and well get the picture of the stage of development. All customized methods are being used along the new implementation route, so it is very easy to maintain life for the next generation. These algorithms are very simple to obtain complete information about the process as they are the natural behavior of any example. This feature allows researchers to apply the natural approach or behaviour of combinations of these. Solving every problem associated with the technical structure can be achieved using these meta-heuristics approaches. According to new versions of the software, these concepts are very favourable to implement and use because the optimized method is using our nature. But the condition is that the researcher must know how to use the technique by natural methods. Whatever input is being given to the system; will this system be able to generate the same output generated by the natural concept? These overall concept requires a very powerful workstation which requires proper connection of systems in a prescribed network with complete details of input work to achieve smooth process of scheduling algorithm. In addition, the use of communication network is commonly designed in a very simple structure of metameterial. Its numerical calculation is also favorable for cost effective cmmunications. Generally, these concepts used in designing of antennas [1]-[5].

Self-made or self-designed methods can only be the concept of any natural thing. Due to this feature, no explanation is required for any method that is going to be used in the new implementation. Genetic methods always begin the process by random number generation of the particle. The output generated for the first time is considered a random solution and then it is applied for the second iteration. One of the most important features of these natural optimization techniques is to avoid local scale and generate consistent results on a large scale. Real problems are under great consideration for developing new solutions. In order to avoid failure and provide solutions to those problems, heuristic algorithms are very capable.

By deriving this motivation lesson, the life principle of grey wolf and honey bee is applied in this paper and the cost factor is calculated as depicted by Figure 2. The quality of leadership is found very high in natural behaviour. Grey wolf optimization (GWO) is also among them. First, it instructs the other grey wolf how to attack the prey. There are four categories of grey wolf to complete the cycle to obtain food. By following the main three steps, this algorithm can be implemented. Influenced by the significance of natural bees, the nature of bees is very effective for gowning the technological world, to capture the ideal solution for upcoming technologies.

Section 1 conclude with the introduction theory for the entire work. Section 2 will explain the things related to the optimization algorithm, and then the next section 3 will write the problem formulation and 
solution regarding the proposed strategy. Further writing done with the section 4 and 5 i.e. implementation results and conclusion respectively.

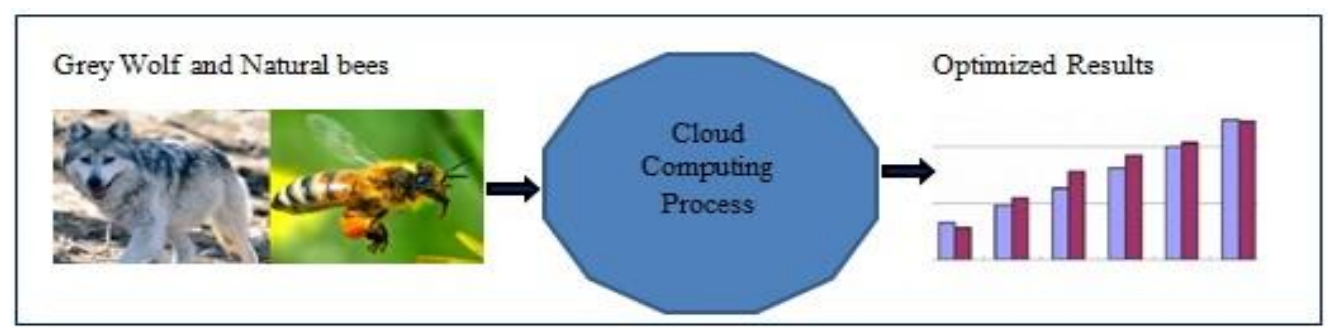

Figure 2. Proposed approach

\section{RELATED WORK}

Various magazines and articles have the most important value in today's market. By getting more publications in the technical field, future working principles of all articles are coming into implementation. Some features are similar to the previous ones and some are in the introduction. Comparisons are being made by applying a new method and results to these characteristics. Natural based techniques produce intelligent results which are very helpful in achieving innovative results. The author uses terms related to natural activities to achieve a world of implementation for real-life problem-solving. The author describes new scheduling using the concept of natural behavior of bees. Due to the heavy workload, new algorithms are required to achieve efficient results. As bees discover food by dancing in different ways, and pass on the information for other bees to follow the shortest path, they are using technology to achieve optimal solutions. Once an optimal function is measured, and then there is no need to recalculate to find the ideal function for other bees [6].

One writer gives a picture of the whole landscape of things of today's innovation. Innovations have been made as a herd optimization technique, using a bee algorithm. By reducing execution time, an effective optimal algorithm is implemented in this paper [7]. The author expressed the evaluation of the gang scheduling algorithm by calculating the cost factor. Concurrent processing is the main goal of this scheduling method, through which multiple tasks can be executed at the same time as well as on different processors. Subtitle functions are performing the same functions as the main functions. In this algorithm one-to-one delimitation is performed between the functions and the processor. This algorithm works most of the time. Most are well aware that organizations are using this concept, for example, Amazon elastic compute cloud (Amazon EC2). In fact the machine can be connected or disconnected from time to time according to their fluctuations. Basic on traditional scheduling algorithms, gang scheduling is implemented by some modification in it [8]. The author publishes an adaptation version in Gang Scheduling. This paper also calculates the load balancing factor, which calculates the starting time frame of the entire stated job. When the queue is empty, the functions will function properly. Due to this knock, the overburden slows down. For example, last job first serve has been transferred to first come first serve [9]. The author speaks for a scheduling method based on load balancing ant colony optimization (LBACO). The primary objective of the system is to divide the tasks into equal percentages to get all machines efficiently the proper function. By obtaining optimized technology LBACO can achieve shorter execution time while processing multiple tasks. This technique is based on the ant colony optimization (ACO) policy [10].

The author demonstrates efficient resource utilization using the mechanism of the max-min-max algorithm [11], [12]. The author illustrates different effective techniques based on herd intelligence. For example, ACO, bee optimization, PSO and GA. In cloud computing, these natural-based intelligence techniques are very effective for finding better results i.e. minimum computation time [13].

An author has analysed about various optimized algorithms in a technical environment. Resource management is a very difficult task; this proposed paper has taken this issue into consideration and played an important role to get a better understanding. The cloud computing field can be improved by considering this issue. Which technique produces what results, which should be in the mind of the writer when developing a new idea for the betterment of the technique, as all methods have their own pros and cons [14]. The author proposed a way to improve energy for private clouds. The combination of the two factors i.e. pre-strength and minimum load has been considered by the author. This idea provides the substantiation of several factors that lead to minimum execution time, energy savings and balance [15]. The author published an algorithm for correlating tasks related to potential resources in the new cloud. By considering two factors of cost and 
performance, the author developed the idea. By amalgamating these two factors, this idea works as the quality of service [16].

The author has created a quality-based methodology that is an improved version of the quality of service (QoS) scheduling algorithm. This paper improves system performance based on time reduction, load averaging and improved latency. By introducing the concept of hybrid technology in the future, this algorithm will work more accurately to achieve efficient execution [17]. The author taps through the ideologies of evolutionism and chromosomal fashion that the Futurist method represents. This applies to the effect of several acts that can be defined as follows: the structure of the initial population, the taxation of the original target generation explanation, the interpretation and spread of the novel, to inspire new inhabitants. Installing new solutions depends on two tasks: crossover and mutation. Now for each operation, the algorithm discovers a new solution (child) and adds the solution to a new population. It selects the solution that is added to the new population using a fixed fitness function [18].

One author proposed the notion of simulated annealing (SA). This is a reality derived from the reinforcement process. In the original proposals, it is the new defective factual derived from the current material by the process of reducing the high temperature which, at the beginning of the process, is gradually initiated through a calling rate. At every level of temperature, the SA algorithm finds a solution [19]. An author provides an ant colony optimization method. It basically works on a probabilistic Bayesian technique that is very helpful for responding to the myriad computational real facts that will be able to reduce the judgment of moral tracks through existing graphs. Artificial ants are used for the persistence of multi-agent devices that are stimulated by the behavior and temperament of natural ants. Pheromone-based communication by biological ants is a commonly used arbitrary criterion. The integration of artificial ants and local search is becoming a method of priority for various optimization tasks related to different types of graphs, such as Internet routing and vehicle routing [20].

The approach of ACO algorithm is determined by the behavior that ants show during the process of searching for food and choosing the shortest route from food to their nest. In addition, whenever an ant receives a passage for food, it downs a chemical substance called pheromone. This material helps to label this pathway which is directed towards other ants. It has been observed that high concentrations of pheromones are found in the minimal-path pathway, and eventually it becomes the preferred route for ants. The ACO algorithm ends with good solutions, but has a very high time complexity. Therefore, ACO may be suitable only for a fairly small number of tasks. Another drawback of using the ACO algorithm is that it results in higher memory usage. In other words, the algorithm requires a high memory to process its computation.

Chemical reaction optimization (CRO) is a newly formulated meta heuristics approach used for optimization. It has been influenced by the type of chemical reactions. A chemical reaction could be defined as a natural process of converting the changeable (or volatile) substances to the stable ones. In an atomic view, a chemical reaction begins with some unsteady molecules with enormous efficiency. The molecules combine with each other through a series of elementary reactions. In the end, they turn into people with the least energy to support their existence. This property is enclosed in CRO, which helps to provide an optimal solution for optimization problems. CRO can be implemented to control problems in both domains as well as discrete as well as continuous domains. Until now CRO has been successfully used in solving various engineering background problems, including quadratic assignment problem, neural network training, multimodal continuous problems and so on. Simulation results suggest that a better performance is established by CRO rather than by an already existing optimization algorithm [21].

The basic concepts of chemical reaction optimization (CRO) have been depicted above. The CRO imitates the chemical reaction operations in which the molecules go through some of the reactions between each other or with the closed container (i.e. the enclosing environment). Every operation provides either one or two new molecules. Each molecule is found to have a unique structure, which depicts a single solution. The algorithm assesses each solution according to the fitness function. The essential features of every molecule are potential energy E and kinetic energy K. The CRO algorithm makes use of $\mathrm{K}$ and $\mathrm{E}$ for accepting or rejecting the new solutions. The authors have suggested some mechanisms to create new solutions for providing an appropriate solution to the task scheduling problem. But the main drawback with these techniques is that they are not found to be good with a large number of tasks. In this paper artificial bees have been developed using the forced behavior of bees. By dividing it into three categories, this method produces good performance. These categories are labor bee, monitoring and active.

The fitness function has been computed to secure the parameter losing information. This algorithm also produces a negative result i.e. high computational cost. So this algorithm can only work on quality based service [22]. Honeybee's algorithm is also effective for load managing factor. Many of the papers are showing the load factor with the combination of cost factor also. In the future, good results will be calculated by the new coming authors. By setting the threshold limit, the load has been measured. An author has shown one drawback also i.e. honeybee's algorithm is not good for migration the process from one to another system when needed [23].

Effective task scheduling algorithm in cloud computing with quality of service alert bees ... (Nidhi Bansal) 
The name suggested for introducing a new publication is "cost-effective load management". By using the concepts of honeybees only, this paper has been designed. By getting the optimal solution from nature, optimal selection of Vm's will be kept. By testing the calculated cost and running, optimal Vm's has been selected to go for main processing. The major drawback of this algorithm is to decrease the overall major aspects of the system [24].

Artificial neural network (ANN) is also coming into the met of task scheduling by using the concept of neural network. Neural network artificially can be developed by changing or updating their weights to get the desired output from the given input. Processing the information artificially can be done with the help of this neural behavior. More accuracy found in these scheduling results, so these are very in use to get more profit by the organization by providing the satisfied outcomes to the user. Artificial maintains the rule of learning to promote the new concept. For walking properly towards the robotic world, keep in the mind some facts those are: Learn from the environment, past experience, and activities of others. "Grind by object" is the channel to enter the range of artificial computers [25]. Many advanced versions of the evolutionary algorithms are in the introduction. It improves the organization of the resources and advancement of the scheduling method. In addition to regular factors, advanced factors come in introduction as trust and reliability [26].

The author has written about the merging technology i.e. hybrid concept. In hybrid technology, one cloud extract the feature of other many clouds contribute more extra benefits to the user by getting assiduities from them. With the combination of one or more flavors, one can make a delicious better for feeding the curious. Using this tag line, by combining more types of cloud, you can develop many useful things that can be a blessing for innovation [27]. The author intentionally presents the caliber of scheduling concepts in this paper. E-learning assignment can also be done only with the help of scheduling techniques.

By choosing the best way of scheduling, important methods must be fitted for the education field. By using mutation and crossover operation of the proposed genetic algorithm, e-learning is performing very well in results [28], [29]. Keeping in mind the recent and valuable research, related work has been written in the paper and is shown in Table 1. With the help of nature, technology is created [30], [31].

Table 1. Comparison

\begin{tabular}{cccc}
\hline Algorithms & Findings & Limitations & Tool used \\
\hline $\begin{array}{c}\text { Majorly nature-based } \\
\text { algorithms }\end{array}$ & $\begin{array}{c}\text { Mainly evaluate the execution } \\
\text { time and } \\
\text { allocation/communication cost }\end{array}$ & $\begin{array}{c}\text { No multi-method techonology } \\
\text { used for scheduling }\end{array}$ & $\begin{array}{c}\text { Commonly Cloudsim and real } \\
\text { cloud plateform of multinational } \\
\text { company }\end{array}$ \\
\hline
\end{tabular}

\section{PROBLEM FORMULATION AND SOLUTION}

The basic need to apply the new idea in a potential new combination research makes the technology much easier for new real and complex tasks to come. In order to get positive results regarding quality in service to overcome the issues which include cost, time, local optima, trust and much more, the concept of nature must accumulate new ideas by adding technology [32], [33]. These concepts are basically established in nature which will be modified by the scheduling concepts. Scheduling problem converted into optimization competition by introducing new optimizing techniques on a daily basis [34], [35]. To choose the best solution factor using mathematics, results of both the optimizing scheduling algorithm have been generated i.e. grey wolf and honeybees.

Cloud computing is basically a technology that examines and figures the capital resources and is transported as a facility above a system in simulated system. A scheduling strategy remains found to be an optimal one if it bears the proficiency to acclimation of mutable atmosphere and nature of jobs. Method employs the features of honey bees and grey wolf life algorithm to plan a working of jobs.

Coming task represents by $\mathrm{T}$ i.e. $\{\mathrm{t} 1, \mathrm{t} 2, \mathrm{t} 3, \mathrm{t} 4, \ldots \mathrm{tn}\}$, MP represents some valid fitness function that must be feasible and capable of producing a result with a possible relational combination execution function. Finally the conclusion will be generated by the optimized results i.e.

$$
\begin{aligned}
& \sum \mathrm{T} \in \mathrm{MP} \\
& \mathrm{MP}=\{\mathrm{mp} \mid \mathrm{mp} \in \mathrm{mp}=\mathrm{R}\}, \mathrm{t}>0\} \text { (where } \mathrm{R} \text { is resource) }
\end{aligned}
$$

In the stated prototypical, the scheduler chooses the capitals so as to designate the works upon the criteria of low performance value. In line with the proposed method (the combination of two), the value of respective datacentre is computed with the help of scout simulated mechanisms. And the fitness point is scaled to examine the fitness point with the minimum expenditure for every datacenter [36], [37]. The less expensive datacentre attains the main fitness point. The figure of simulated engines is determined by the 
computation of datacentres. The number of virtual machines is always found to be similar to the overall measurement of datacentres. All the jobs in the purposive system is found to keep the ensuing presumptions:

a) All jobs are non-periodic: entrance time of jobs is recognized a main key factor. The organized time of a job is equivalent to its entrance time.

b) Cost and Time of capitals: resources are abandoned on the actions that are being performed on every single unit of service. So that one may extent the charges of requests precisely, each distinct usage of the various capitals like RAM cost, and bandwidth cost. Essentially be ensured to be evaluated. In accordance with the type of task, every task has its own finishing time.

c) Non-protective tasks: every task in the system are assumed to be non-protective. It means that the processor cannot leave the task unless and until the task has successfully completed its execution.

Referenced research papers are telling all about the innovation, implementation of new ideas. Whatever results in this toolkit gives, the same results can be generated by the real cloud server? Introducing the new factors i.e. trust is in communication for creating the computing world. Artificial intelligence techniques also get in use to implement task scheduling algorithms. Hiding and securing the data is a very crucial term in these days, every author wants to secure the data [38], [39]. By applying the secure authentication method, the task scheduling algorithm will be created. This factor will take of its own importance in the developing field.

\section{PROPOSED ALGORITHM}

Optimized honeybees algorithm has been proposed to overcome the existing problem or issues from the common lifestyle through developing harmonious algorithm with some advanced and effective results. An advanced algorithm for bees and grey wolf is decribed here for getting deep analysis and understanding. The defined algorithm shows the process of execution for both concepts, as shown in the flow chat in this section.

\subsection{Honeybees}

If we search for an insect that could provide us useful and intelligent optimization technique, then the best and most studied insect is honeybee. It is useful in providing an effective solution for complex and computational problems. As like the natural behavior of bees, there are two models that are studied. The first model is based on foraging behavior. It is further subdivided into models: i) food foraging and ii) nest site inquiring.

Another model consists of nuptial behavior in the bee society. The term bee colony refers to the behavior and mode of communication of bee. For solving the problems such as the travelling salesman problem, graph coloring food source searching model is being used. As per many suggestions and proposals from time to time, resource scheduling is getting the main role in the cloud via Bee soul Algorithm for a diverse atmosphere. As we know that some tasks are found to be central processing unit (CPU) severe, few are i/o rigorous, specific wants extra retention and particular entail extraordinary processing locks. Therefore, the process of allocating the tasks on their own servers in an improved way is done by bee's life algorithm. Here, we are basically dealing with food foraging model. The utilization of datacentres is being maximized by the computation of fitness function. After the calculation of fitness function, the fitness value needs not to be found again. Honey bees soul algorithm could be explained as:

a) The first phase starts with booting the population (n) i.e., the count of virtual machine (Vms) is same as Realizable value. The target sample population of VMs behaves as selected scout bees. The process of selecting individual datacentre (DCs) for VMs is done stochastically. These selected scouts are indiscriminately within the available VM location.

b) All DCs found its characteristics (Dchs). Valuable features of DCs support VMs contributing to conserve reserve resources at certain superiority threshold values. Each DC includes the following features: memory, repository, bandwidth, and effective execution time.

c) Based on the above constraints, the fitness point is assessed. The fitness point is calculated to examine how many capital resources are distributed through particular DCs. The time taken to exceed the cost captured by resources for accomplishment is also calculated. The fitness point of every particular DC is estimated.

d) Great scouts and great VMs being chosen. Now with the best Vms, the best VMs are being nominated. The DC that distributes better capital resources according to the requirement of the works is selected. The VM of such DCs behaves as the selected scout VM from the selected scout Vms.

e) The well-resevered resource that is given to DC is found to be an exceptional fitness point. And now it is named for the implementation of DC work. After the selection process for EBEST DC, the target tasks will get assigned as the top priority to complete the proposed tasks. Permanent tasks on the Target Scout VMS are referenced arbitrarily. 
f) The outstanding VM (VM - Best) and VMS Residual (Best - Ebest) novel residents are being prepared, and the process resumes.

g) The Honey Bees algorithm has applications for composite and computation problems. This algorithm provides optimal and general solutions. It is based on jobs scattered between active properties.

Therefore, it improves the overall performance of tasks and increases the usability of all cloud computing facilities and is shown in Figure 3. In this, rescheduling is done following the traditional concept with some progress. Some problems or shortcomings occur from time to time, so the optimized version needs to be launched.

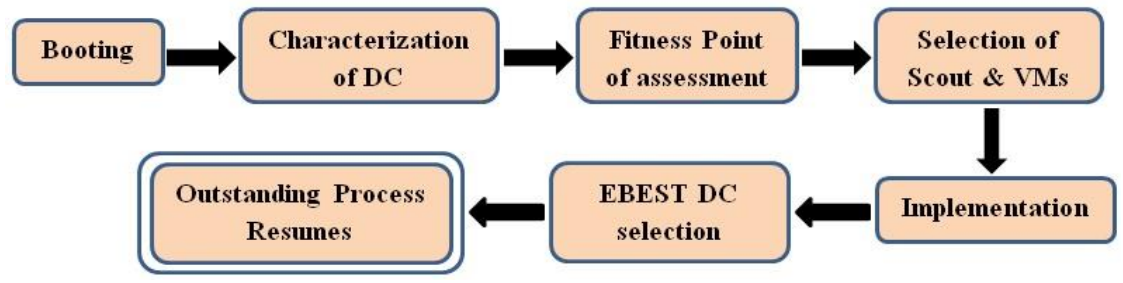

Figure 3. Honeybees' algorithm flow chart

\subsection{GWO}

The GWO strategy proceeds by following steps:

a) General chain of command: inner's grey wolf plays the important role to get the prey, then continue ling inner lever plays a role with minimum effort then outer wolves passing the information to most outer wolves to get the prey.

b) Begird prey: grey wolf begins the prey to get hunt, by using the distance equations of mathematics. By getting the distance value, wolves will be able to make the correct position from where wolves can get the prey easily. Kill the prey: prey is only surrounded by the inner's wolves, so inner wolves are always live in the danger. The number of inner wolves is always three, not more than. Due to this, we get the three positions for the solution.

The grey wolf life algorithm can be explained as follows:

- The first stage starts with booting the population (n) i.e., the count of Vms is the same as the exact value. This goal of VM treats citizenship as grey wolf. The process of election of exclusive DC to VM is done casually. These target VMs are fortunately switched to probe locations (obtainable Dcs).

- $\quad$ Repeat the procedure till all steps are completed

- Now to get the nearest position of prey, construct the dots as a cluster. Larger fitness points are found in DC provided better resources. And now it is nominated for a good performance of DC work. Following the process of selecting the abest DC, several activities for the simulation are collaborated with that DC. Outlast tasks are addressed incidentally on the Scout VMS.

- Re-compute the position of each grey wolf to find the best-fitted value.

- Until the situation changes.

The presented paper embodies the experimentation and rich system architecture to correlate the resources required on the virtual machine to meet the buyer's demands. Absolute satisfaction depends on many factors and characteristics as shown in Figure 4. As such, user satisfaction percentages improved by writing the complete paper with numerical exercises for the cost and time of task allocation on the virtual machine.

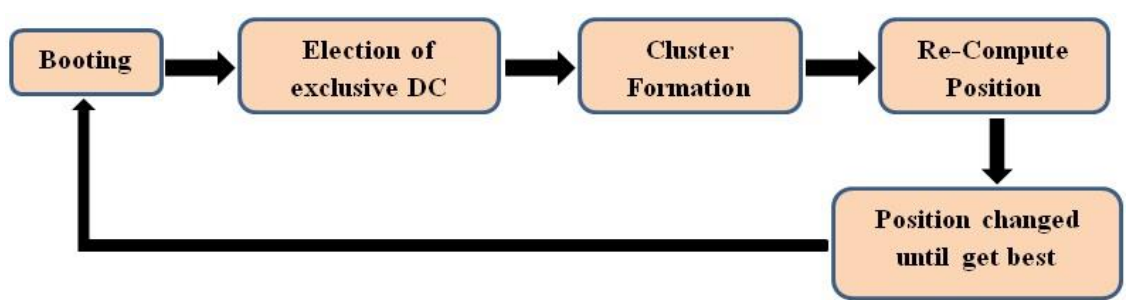

Figure 4. GWO algorithm flow chart 


\subsection{Pros and cons}

Generally, task scheduling performs well in terms of simple and fast execution. A good task scheduling mainly focuses on the resources required, efficient memory usage, minimum time, and cost. Its main concern is always the performance of the system. All these important facts may convert into real facts with the help of natural phenomena with proven results and conclusions. In addition to the theory section, this optimized approach is implemented by the actual implementation and the graphs are shown in the section 4 .

\subsection{Implementation}

Regardless of the scheduling, the mechanism's approach is divided into different directions. Due to more directions, the curse of dimension also comes in the picture. To reduce dimensions, much new mathematics has been used to avoid this mobility. This system also becomes a syllabic topic for machine learning. Furthermore, the goal of cloud computing is difficult to bend to the trademark of cloud automation. Proceed with this section, implementation, and results of natural living being optimized algorithms showing the best results here. The well-known simulator i.e., cloudsim is doing simulation for the proposed model. Specifications of using a laptop to do the simulations are i3 processor with 2 GB ram and 64-bit windows 7 operating system. The outcome of the proposed comparison of both the techniques, in terms of allocation cost shows in this section. By definition, the fitness situation is in practice and can determine the virtue of every creature from many samples. From the root of mathematics, fitness tasks can be measured.

A function can only be generated from the math function. To compare different innovation techniques in the most prominent manner, the highest rank functions used to calculate the fruitful values. The fitness point of respectable resource is calculated by the factor allocation cost. The formula for determine fitness value is comes from:

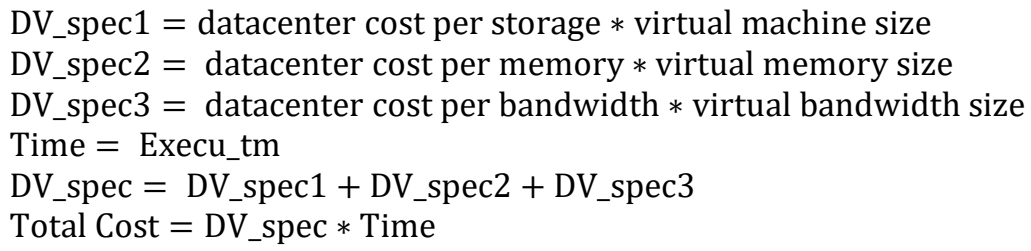

Where, DV_Spec are serials with the specification of datacenter and virtual machine, dah $=$ datacenter specifications, banwd = datacenter/virtual machine's bandwidth, memory $(\mathrm{MEM})=$ datacenter/virtual machine's random-access memory (RAM) and Execu_tm $=$ computation execution time for $\mathrm{n}$ tasks on different data centers. Str $=$ Storage cost of the datacenter is dealing with virtual machine's repository; the memory usage of a datacenter is dealing with virtual machine's RAM usage and bandwidth's cost of datacenter is dealing with virtual machine's bandwidth consumption is computed. Then, do summations for the particular costs and in inference, do multiplication with the estimated execution time with reference to its description on the notable datacenter.

The accurate configuration will always be correct to get the correct results. So, make the system trustable that will be able to rectify all the future coming problems or error. Table 2 is depicting the whole setup for executing the proposed method. Right measurements and guesses are ever being supported for promoting the present as well as future things. So, keep faith in the probable shapes.

Table 3 and Table 4 are showing the clarified results between the calculated estimation of the allocation cost by running the proposed algorithm in cloudsim toolkit. Experimental results showed that in all instances with less than 100 nodes, the bee system produced the optimal solution in a very short time as shown in Figure 5. Optimized algorithm is always appropriate for developing the new technology towards the growth of our technological India as shown in Figure 6.

Table 2. Experimental setup

\begin{tabular}{ccc}
\hline Variable & Behaviour Speed & Value \\
\hline Data storage & Center, Host, Virtual machine & $2,1,30$ \\
Virtual Machine & MIPS, PE, RAM, BW, Type & $4000,1,512 \mathrm{MB}, 1000000^{*}$, Space shared \\
Cloudlets & Number, Length & $30,40000 \mathrm{MI}$ \\
PE & & 1 \\
\hline
\end{tabular}

Table 3. Basic and optimized QoS

\begin{tabular}{cc}
\hline HoneyBees & Basic Bees \\
\hline $12,486.4$ & 24,496 \\
$4,682.4$ & 12,248 \\
\hline
\end{tabular}

Table 4. Optimized results for QoS

\begin{tabular}{cc}
\hline Optimized Bees & GWO \\
\hline $6,243.2$ & $1,560.8$ \\
$4,682.4$ & 6,124 \\
\hline
\end{tabular}




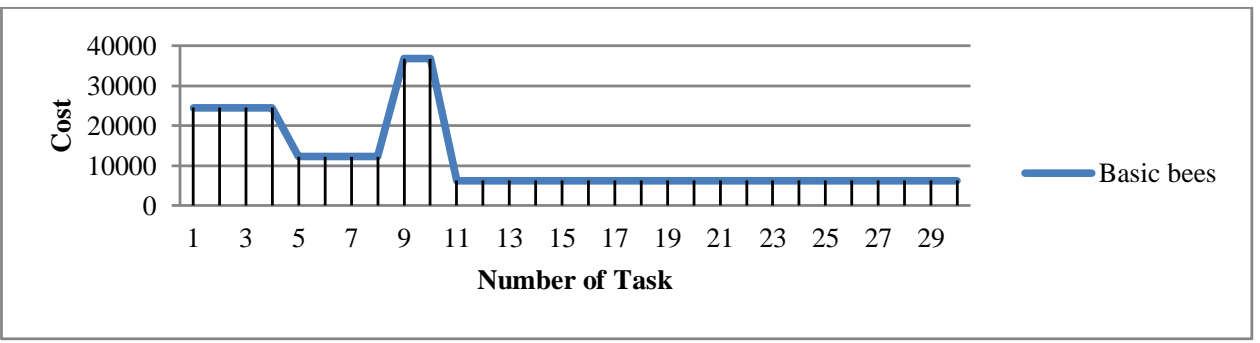

Figure 5. Cost performance of basic bees

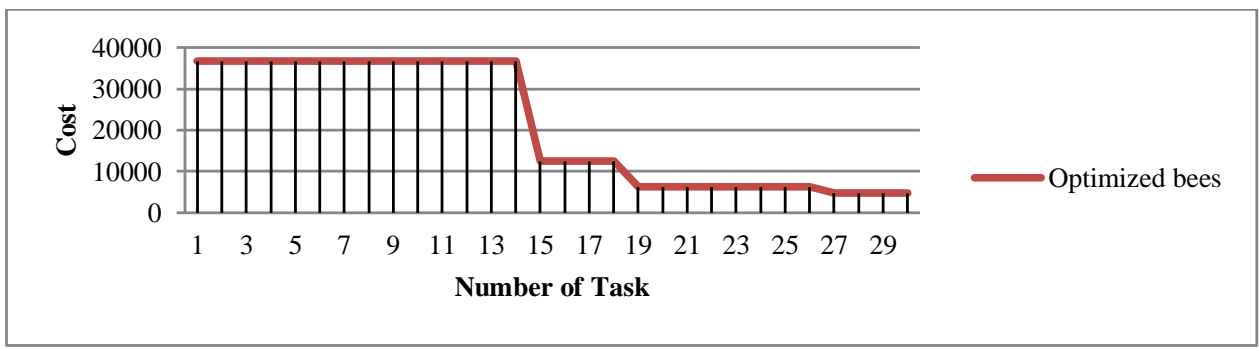

Figure 6. Cost performance of optimized bees

In the proposed work, the tables and graphs presented attempted to overcome the limitation by performing complex tasks in real time on the designed network. With the available data and algorithms, the proposed algorithm is good for comparing results with existing ones and emerging results are shown from the data. The prediction is made by our own intelligence as a proven principle by analyzing the actual data performance on the designing implementation setup.

The natural behavior of living animals is observed and applied in technology by combining two natural behaviors in the form of algorithms to achieve the goal in the study. This has been accomplished with the results shown by Figure 7. Learning is also enhanced by incorporating nature-based events to achieve greater accuracy in the results.

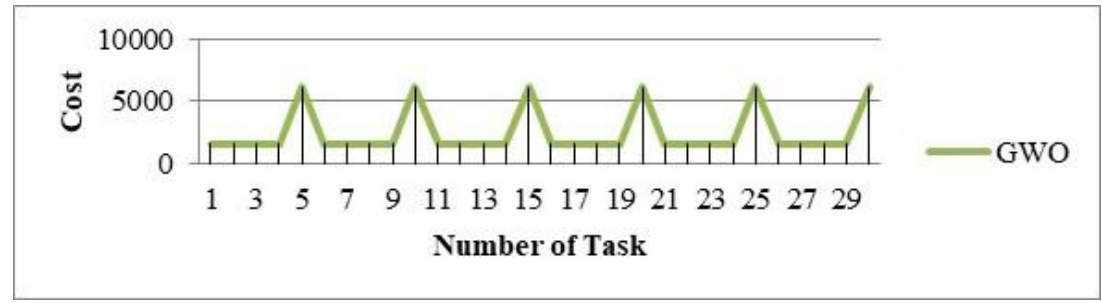

Figure 7. Cost performance of GWO

\section{CONCLUSION AND FUTURE WORK}

The Cloud computing model is used for developing new innovative ideas in all aspects. Because of this positive result, cloud computing has become the most valuable work tool to achieve all the achievements. In this paper, a comparison is made between natural and optimized algorithms to solve the task scheduling problem in cloud computing to enhance systems and technology. The proposed method able to solve the problem of tasks for real and dynamic nature, i.e. coming up with effective results by implementing an optimized algorithm. Lastly, the paper would like to assure that, the cost-effective algorithms ensuring for the security of user's data by $60 \%$. As its also mentioned in the recent scenario of global benchmarking that is the quality must be a very important attribute for every organized process. For marketers, this concept has proven to be very efficient thanks to the continuous improvement of task scheduling algorithms and the requirements of today's market. From the results, the solution of the formulation is stored. Because of this, the new results are very valuable for creating a new solution for more problems in real-world applications. For the foreseeable future, more techniques for new generalizations can be generated using hybrid cloud computing. 
Future worlds could also recur by achieving a combination of more than two hybrid clouds such as fuzzybased solutions. Natural facts may be a better way to improve our world in the digital world.

\section{ACKNOWLEDGEMENTS}

Thanks to Dr APJ Abdul Kalam University for providing a good platform for my higher studies.

\section{REFERENCES}

[1] M. Alibakhshikenari et al., "A Comprehensive Survey of "Metamaterial Transmission-Line Based Antennas: Design, Challenges, and Applications," inIEEE Access, vol. 8, pp. 144778-144808, 2020, doi: 10.1109/ACCESS.2020.3013698.

[2] M. Alibakhshikenari et al., "A Comprehensive Survey on Various Decoupling Mechanisms With Focus on Metamaterial and Metasurface Principles Applicable to SAR and MIMO Antenna Systems,” in IEEE Access, vol. 8, pp. 192965-193004, 2020, doi: 10.1109/ACCESS.2020.3032826.

[3] M. Alibakhshikenari, B. S. Virdee, A. Ali, and E. Limiti, "Extended aperture miniature antenna based on CRLH metamaterials for wireless communication systems operating over UHF to C-band," in Radio Science, vol. 53, no. 2, pp. 154-165, Feb. 2018, doi: 10.1002/2017RS006515.

[4] M. Alibakhshikenari, B. S. Virdee, C. H. See, R. A. Abd-Alhameed, F. Falcone, and E. Limiti, "High-isolation leaky-wave array antenna based on CRLH-metamaterial implemented on SIW with \pm 30 o frequency beam-scanning capability at millimetre-waves," Electronics, vol. 8, no. 6, 2019, doi: 10.3390/electronics8060642.

[5] M. Alibakhshikenari et al., "Antenna mutual coupling suppression over wideband using embedded periphery slot for antenna arrays," Electronics, vol. 7, no. 9, 2018, doi: 10.3390/electronics7090198.

[6] R. Pradeep and R. Kavinya, "Resource Scheduling in Cloud using Bee Algorithm for Heterogeneous Environment," International Organization of Scientific Research-Journal of Computer Engineering, vol. 2, no. 4, pp. 15-16, 2012.

[7] S. Bitam, "Bees Life Algorithm for Job Scheduling in Cloud Computing," Proceedings of the third international conference on communications and information technology, vol. 7, no. 8, 2012, pp. 186-191.

[8] I. A. Maschakis and H. D. Karatza, "Evaluation of Gang Scheduling Performance and Cost in a Cloud Computing System," The Journal of Supercomputing, vol. 59, no. 2, pp. 975-992, 2012, doi: 10.1007/s11227-010-0481-4.

[9] S. Singh, "Performance Optimization in Gang Scheduling in Cloud Computing," International Organization of Scientific Research - Journal of Computer Engineering, vol. 2, no. 4, pp. 49-52, 2012.

[10] K. Li, G. Xu, G. Zhao, Y. Dong, and D. Wang, "Cloud Task Scheduling Based on Load Balancing Ant Colony Optimization," 2011 Sixth Annual Chinagrid Conference, 2011, pp. 3-9, doi: 10.1109/ChinaGrid.2011.17.

[11] C. L. Hung, H. H. Wang, and Y. C. Hu, "Efficient Load Balancing Algorithm for Cloud Computing Network," International Conference on Information Science and Technology, 2012, pp. 251-253.

[12] H. Chen, F. Wang, N. Helian, and G. Akanmu, "User-priority guided Min-Min scheduling algorithm for load balancing in cloud computing," 2013 National Conference on Parallel Computing Technologies (PARCOMPTECH), 2013, pp. 1-8, doi: 10.1109/ParCompTech.2013.6621389.

[13] S. Bilgaiyan, S. Sagnika, and M. Das, "An Analysis of Task Scheduling in Cloud Computing using Evolutionary and Swarm based Algorithms," International Journal of Computer Applications, vol. 89, no. 2, pp. 11-18, 2014.

[14] F. Mohammadi, S. Jamali, and M. Bekravi, "Survey on Job Scheduling Algorithms in Cloud Computing," International Journal of Emerging Trends and Technology in Computer Science, vol. 3, no. 2, pp. 151-154, 2014.

[15] J. Li, J. Peng, Z. Lei, and W. Zhang, "An Energy- Efficient Scheduling Approach Based on Private Clouds," Journal of Information \& Computational Science, vol. 8, no. 4, pp. 716-724, 2011.

[16] S. Selvarani and G. S. Sadhasivam, "Improved cost-based algorithm for task scheduling in cloud computing," 2010 IEEE International Conference on Computational Intelligence and Computing Research, 2010, pp. 1-5, doi: 10.1109/ICCIC.2010.5705847.

[17] X. Wu, M. Deng, R. Zhang, B. Zeng, and S. Zhou, "A Task Scheduling Algorithm based on QoS-driven in Cloud Computing," Procedia Computer Science, vol. 17, pp. 1162-1169, 2013, doi: 10.1016/j.procs.2013.05.148.

[18] Y.-K. Lin and C. S. Chong, "Fast GA-based project scheduling for computing resources allocation in a cloud manufacturing system," J. Intell. Manuf., vol. 28, no. 5, pp. 1189-1201, 2017, doi: 10.1007/s10845-015-1074-0.

[19] N. Shivasankaran, P. S. Kumar, and K. V. Raja, "Hybrid sorting immune simulated annealing algorithm for flexible job shop scheduling," Int. J. Comput. Intell. Syst., vol. 8, no. 3, pp. 455-466, 2015, doi: 10.1080/18756891.2015.1017383.

[20] R. Achary, V. Vityanathan, P. Raj, and S. Nagarajan, "Dynamic job scheduling using ant colony optimization for mobile cloud computing," Intelligent Distributed Computing, pp. 71-82, 2015, doi: 10.1007/978-3-319-11227-5_7.

[21] Y. Xu, K. Li, L. He, L. Zhang, and K. Li, "A Hybrid Chemical Reaction Optimization Scheme for Task Scheduling on Heterogeneous Computing Systems," in IEEE Transactions on Parallel and Distributed Systems, vol. 26, no. 12, pp. 3208-3222, Dec. 2015, doi: 10.1109/TPDS.2014.2385698.

[22] D. Karaboga, "An idea based on honey bee swarm for numerical optimization," Technical Report TR06, Computer Engineering Department, Engineering Faculty, Erciyes University, Turkey, 2005.

[23] W. Hashem, H. Nashaat, and R. Rizk, "Honey Bee Based Load Balancing in Cloud Computing," KSII Transactions on internet and information systems, vol. 11, no. 12, pp. 5694-5711, 2017, doi: 10.3837/tiis.2017.12.001.

[24] Y. S. Sheeja and S. Jayalekshmi, "Cost effective load balancing based on honey bee behaviour in cloud environment," 2014 First International Conference on Computational Systems and Communications (ICCSC), 2014, pp. 214-219, doi: 10.1109/COMPSC.2014.7032650.

[25] D. B. Karunakar and G. L. Datta, "Controlling Green Sand Mould Properties Using Artificial Neural Networks and Genetic Algorithms-A Comparison," Applied Clay Science, vol. 37, no. 1-2, pp. 58-66, 2007, doi: 10.1016/j.clay.2006.11.005.

[26] J-T. Tsai, J-C. Fang, and J-H. Chou, "Optimized Task Scheduling and Resource Allocation on Cloud Computing Environment Using Improved Differential Evolution Algorithm,” Computers \& Operations Research, vol. 40, pp. 3045-3055, 2013, doi: 10.1016/j.cor.2013.06.012.

[27] M. Malathi, "Cloud Computing Concepts," 3rd International Conference on Electronics Computer Technology (ICECT), vol. 6, 2012, pp. 236-239. 
[28] O. Morariu, C. Morariu, and T. Borangiu, "A Genetic Algorithm for Workload Scheduling in Cloud Based E-Learning," Proceedings of the 2nd International Workshop on Cloud Computing Platforms (CloudCP), no. 5, 2012, pp. 1-6, doi: $10.1145 / 2168697.2168702$.

[29] N. Bansal and A. K. Singh, "Trust for Task Scheduling in Cloud Computing Unfold it through Fruit Congenial," Networking Communication and Data Knowledge Engineering, vol. 4, pp. 41-48, 2017, doi: 10.1007/978-981-10-4600-1_4.

[30] N. Bansal, A. Awasthi, and S. Bansal, "Task scheduling algorithms with multiple factor in cloud computing environment," Information Systems Design and Intelligent Applications, vol. 433, pp. 619-627, 2016, doi: 10.1007/978-81-322-2755-7_64.

[31] N. Bansal, A. Maurya, T. Kumar, M. Singh, and S. Bansal, "Cost performance of Qos driven task scheduling in cloud computing," Procedia Computer Science, vol. 57, pp. 126-130, 2015, doi: 10.1016/j.procs.2015.07.384.

[32] N. Bansal and M. Dutta, "Performance evaluation of task scheduling with priority and non-priority in cloud computing," 2014 IEEE International Conference on Computational Intelligence and Computing Research, 2014, pp. 1-4, doi: 10.1109/ICCIC.2014.7238289.

[33] N. Bansal and A. K. Singh, "Grey Wolf Optimized Task Scheduling Algorithm in Cloud Computing," Frontiers in Intelligent Computing: Theory and Applications. Advances in Intelligent Systems and Computing, vol. 1013. pp. 137-145, 2020, doi: 10.1007/978-981-32-9186-7_16.

[34] N. Bansal, K. P. Jayant, P. Singh, and S. Pandey, "Intelligent Extension with Smart Connections using Bluetooth with IoT," 2021 5th International Conference on Trends in Electronics and Informatics (ICOEI), 2021, pp. 435-439, doi: 10.1109/ICOEI51242.2021.9452859.

[35] M. Alibakhshi-Kenari, M. Movahhedi, and H. Naderian, "A new miniature ultra wide band planar microstrip antenna based on the metamaterial transmission line," 2012 IEEE Asia-Pacific Conference on Applied Electromagnetics (APACE), 2012, pp. 293-297, doi: 10.1109/APACE.2012.6457679.

[36] F. Arena and G. Pau, "An overview of big data analysis," Bulletin of Electrical Engineering and Informatics, vol. 9, no. 4, pp. 1646-1653, 2020, doi: 10.11591/eei.v9i4.2359.

[37] Y. Zhang, S. Wang, H. Xia, and J. Ge, “A Novel SVPWM Modulation Scheme," 2009 Twenty-Fourth Annual IEEE Applied Power Electronics Conference and Exposition, 2009, pp. 128-131, doi: 10.1109/APEC.2009.4802644.

[38] M. Giliberto, F. Arena, and G. Pau, "A fuzzy-based Solution for Optimized Management of Energy Consumption in e-bikes," Journal of Wireless Mobile Networks, Ubiquitous Computing, and Dependable Applications (JoWUA), vol. 10, no. 3, pp. 45-64, 2019, doi: 10.22667/JOWUA.2019.09.30.045.

[39] H. Aljarajreh, D. D. -C. Lu, Y. P. Siwakoti, R. P. Aguilera, and C. K. Tse, “A Method of Seamless Transitions Between Different Operating Modes for Three-Port DC-DC Converters," in IEEE Access, vol. 9, pp. 59184-59195, 2021, doi: 10.1109/ACCESS.2021.3073948.

\section{BIOGRAPHIES OF AUTHORS}
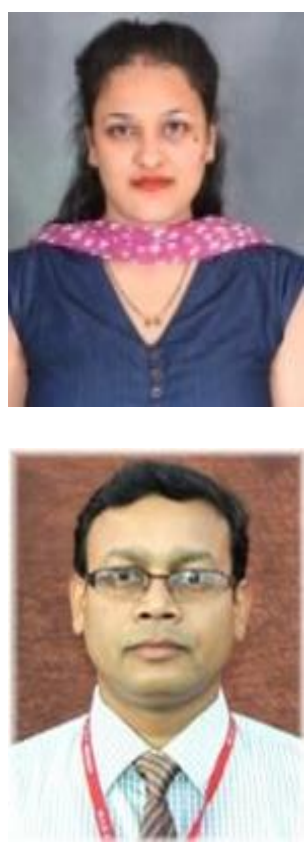

Nidhi Bansal (iD 8d SC P born in December 1990, Meerut city. She is pursuing PhD with CSE branch from AKTU (UPTU) Lucknow. She is working as an Assistant Professor from 2014 after M.E. from NITTTR Chandigarh. Her current research interests include Scheduling algorithms, Cloud computing technologies, IoT concepts, Machine Learning algoithms and its applications. She can be contacted at email: nidhi18jul@mail.com.
Dr. Ajay Kumar Singh (iD 8d SC P born in 1974 at Dhanbad (Jharkhand). He had done B.E (Computer Science \& Engg.) from Kumaon Engineering College, M. Tech (I.T) Allahabad, Ph. D (Computer Science \& Engg.) Jaypee University of Information Technology. Work Experience: He had been in different institution/university like RGEC, Meerut, (U.P), Sir PSU, Bhatewar, Udaipur, Rajasthan, JUIT, Waknaghat, Solan (H.P), MITS, Lakshmangarh, Sikar, Rajasthan, N.I.T Kurukshetra (Haryana), SSI Ltd. (Delhi). Now he is working with MIET, Meerut, U. P. He has published more than 60 papers in reputed Journals/Conferences. He can be contacted at email: ajay41274@gmail.com. 